\title{
Using Dafny to Solve the VerifyThis 2021 Challenges $^{*}$
}

\author{
Marie Farrell \\ marie.farrell@mu.ie \\ Maynooth University \\ Maynooth, Co. Kildare, Ireland
}

\author{
Conor Reynolds \\ conor.reynolds@mu.ie \\ Maynooth University \\ Maynooth, Co. Kildare, Ireland
}

\author{
Rosemary Monahan \\ rosemary.monahan@mu.ie \\ Maynooth University \\ Maynooth, Co. Kildare, Ireland
}

\begin{abstract}
This paper provides an experience report of using the Dafny program verifier, at the VerifyThis 2021 program verification competition. The competition aims to evaluate the usability of logic-based program verification tools in a controlled experiment, challenging both the verification tools and the users of those tools. We present the two challenges that we tackled during the competition and discuss our solutions. As a result, we identify strengths and weaknesses of Dafny in the verification of relatively complex algorithms, and report on our experience of applying Dafny in this setting.
\end{abstract}

\section{CCS CONCEPTS}

- Software and its engineering $\rightarrow$ Formal software verification.

\section{KEYWORDS}

Deductive Verification, VerifyThis, Dafny, Verification Challenges

ACM Reference Format:

Marie Farrell, Conor Reynolds, and Rosemary Monahan. 2021. Using Dafny to Solve the VerifyThis 2021 Challenges. In Proceedings of the 23rd ACM International Workshop on Formal Techniques for Java-like Programs (FTffP '21), fuly 13, 2021, Virtual, Denmark. ACM, New York, NY, USA, 7 pages. https://doi.org/10.1145/3464971.3468422

\section{INTRODUCTION}

This paper provides an experience report of using the Dafny program verifier, at the VerifyThis 2021 program verification competition. During the competition our team, comprised of one post-doc and one PhD student tackled two of the three challenges posed ${ }^{1}$ using the Dafny program verifier [14].

The competition examined both the verification tools and the users of those tools. As a result, we were able to identify some strengths and weaknesses in Dafny, as well as harness the opportunity to educate ourselves on applying this formal verification tool to relatively complex algorithmic problems.

This paper is structured as follows. Section 2 briefly summarises the VerifyThis competition series and the Dafny program verifier. Section 3 describes and discusses two of the competition challenges and our corresponding Dafny solutions, constructed during the

${ }^{*}$ This work is partially funded by the Irish Research Council: GOIPG/2019/4529. ${ }^{1}$ Authors Farrell and Reynolds participated as the "Maynooth University" team.

\section{(c) (9)}

This work is licensed under a Creative Commons Attribution 4.0 International License.

FTffP '21, fuly 13, 2021, Virtual, Denmark

(c) 2021 Copyright held by the owner/author(s).

ACM ISBN 978-1-4503-8543-5/21/07.

https://doi.org/10.1145/3464971.3468422 competition period. In Section 4 we discuss our experience and outline some of the strengths and weaknesses of our chosen tool for these challenges. Finally, Section 5 concludes.

\section{BACKGROUND}

In this section, we briefly summarise the structure of the VerifyThis competition series and describe the verification tool that we used.

\subsection{VerifyThis Competition}

VerifyThis is a series of program verification competitions that has been held at FoVeOOS2011 [2], FM2012 [7], Dagstuhl Seminar 14171 (2014), ETAPS 2015-2019 [4, 8-11] and 2021, inspired by the VSTTE 2010 competition [12]. VerifyThis aims to evaluate the usability of logic-based program verification tools in an easily repeatable, controlled experiment, and to bring together and encourage discussion between those interested in formal verification. The competition challenges are described in a combination of natural language and pseudo code. Participants must formalise the requirements, implement a solution, and formally verify the implementation for adherence to the specification. There are no restrictions on the programming language or verification technology used.

The correctness properties posed in the problems focus on the input-output behaviour of programs. Solutions are judged for correctness, completeness, and elegance. Typically, VerifyThis is a 2 -day event. The on-site competition on day 1 consists of three 90 minute challenges which are released consecutively. On day 2 the jury discusses the solutions with each team participating, in parallel all participants present and discuss their solutions and their different approaches. VerifyThis 2021 was unusual because it took place as an online-only event (due to COVID-19). The three challenges were issued concurrently, with a 24-hour period allocated for participants to attempt solutions. Challenges were intentionally made more difficult than those for the on-site competition because of the extended time period allowed. Day 2 then consisted of participants short online presentations of solutions. Twenty-three teams submitted solutions, three of which used Dafny as their main tool.

\subsection{Dafny}

Dafny is a programming language with a program verifier, used to statically verify functional program correctness, allowing users to define specification constructs e.g. pre-/post-conditions [14]. For verification, programs are translated into the Boogie intermediate verification language [1] and then the Z3 automated theorem prover discharges the associated proof obligations [3].

Figure 1 shows the structure of a method with specification constructs in Dafny. Pre- and post-conditions are indicated by the requires (line 2) and ensures (line 4) clauses respectively. The modifies clause (line 3 ) allows the user to specify a frame 


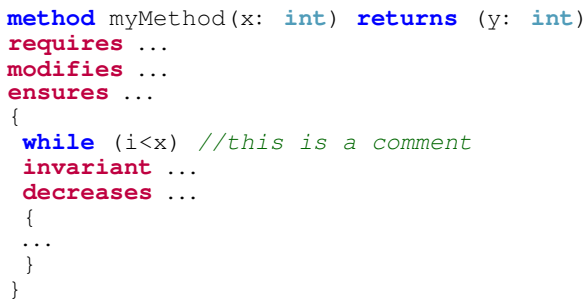

Figure 1: The basic structure of a method with specification constructs in Dafny.

condition that denotes the set of memory locations that are allowed to be modified. Loop invariants (line 7) are used to reason about the correctness of loops and provide support for verifying related postconditions. The loop variant, identified as a decreases clause (line 8), is used to prove termination. Dafny supports specificationonly ghost code, which is erased in the resulting executable and is only available for use by the verifier. Dafny has been used in previous VerifyThis competitions $[8,11]$ and also in larger systems $[5,6]$. We used Dafny as part of Visual Studio Code $[13]^{2}$.

\section{THE CHALLENGES}

VerifyThis 2021 consisted of three verification challenges. Of these three, we were able to partially complete two challenges during the allotted competition time. This section summarises and describes our efforts. Full source code is available in our repository ${ }^{3}$.

\subsection{Challenge 1: Lexicographic Permutations}

"This challenge considers an algorithm that takes any sequence $A$ as input, and enumerates all possible permutations of $A$. Moreover, it enumerates these permutations in sorted (lexicographic) order."

The basic algorithm that was given to competitors is provided in Figure 2. The verification tasks are summarised as follows ${ }^{4}$ :

Task 1.1: Verify that 'next' is memory-safe.

Task 1.2: Verify that 'next' terminates for every input.

Task 1.3: Verify that any changes on 'a' performed by 'next' are permutations.

Task 1.4: Verify that, if 'next(a)' returns false, then 'a' is left unmodified and is indeed the last permutation in the sequence.

Task 1.5: Verify that, if 'next(a)' returns true, then 'a' is modified to be the proper next permutation in the sequence.

Task 1.6: Verify that 'permut' is memory-safe.

Task 1.7: Verify that 'permut' terminates for every input.

Task 1.8: Verify that any permutation reported by 'permut' is unique.

Task 1.9: Verify that 'permut(a)' reports all permutations of 'a'.

Task 1.10: Verify that 'permut' outputs all permutations in lexicographic order.

We specified numerous predicates in Dafny to help with verifying properties about the alogorithm. These are summarised in Figure 3:

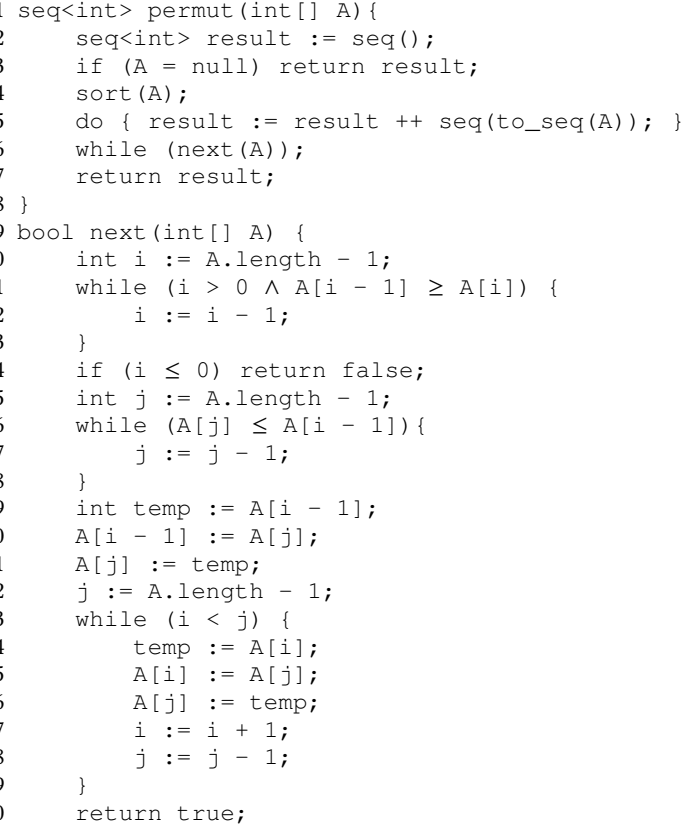

Figure 2: This pseudo code was given to the competitors as part of the Challenge 1 description. Note that ' ++ ' denotes sequence concatenation.

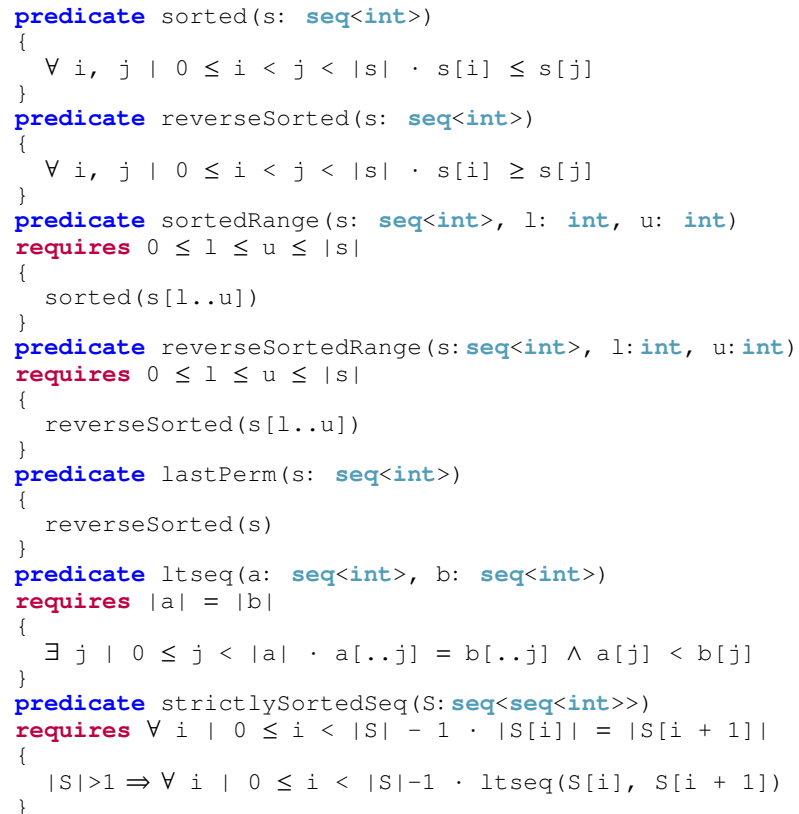

Figure 3: Predicates in Dafny for Challenge 1.

\footnotetext{
${ }^{2}$ Dafny version 3.1.0.

${ }^{3}$ https://github.com/mariefarrell/MUVerifyThis2021.git

${ }^{4}$ https://www.pm.inf.ethz.ch/research/verifythis/Challenges.html
} 
Lines 1-18: The sorted and reverseSorted predicates are used to describe what it means for the sequence to be sorted in ascending and descending order respectively. Based on these the sortedRange and reverseSortedRange predicates consider ascending and descending sortedness, respectively, given a range of the input sequence.

Lines 19-22: The last Perm predicate allows us to identify when we have reached the final permutation which, since it is the last in the lexicographic order, will be reversesorted. This is particularly useful when verifying Task 1.4.

Lines 23-32: The ltseq predicate takes two sequences of the same size as input and checks whether one is less than, in terms of the lexicographic ordering, than the other. This predicate is used by the strictlysortedSeq predicate which determines whether an input sequence of sequences is in lexicographical order. This is used to verify Task 1.10 .

Figure 4 contains our Dafny implementation of the next and permut methods as outlined in Figure 2.

Lines 1-5: These pre- and post-conditions verify Tasks 1.3 , 1.4 and 1.5. The modifies clause (line 2) relates to the frame condition and allows us to modify the input array of integers. This supports memory safety in Task 1.1 by specifying the memory locations (a) that are allowed to be modified. We verify that the modified array contains the same elements as the input array using Dafny's multiset construction on line 3, this corresponds to Task 1.3. The post-condition on line 4 corresponds to the verification of Task 1.4 which specifies that if next returns false then a is unmodified and is the last permutation in the sequence. In contrast, we partially verify Task 1.5 via the post-condition on line 5 which specifies that, if next returns true then $a$ is modified to be the proper next permutation in the sequence. We partially verify this property because we do not give sufficient treatment to the proper next permutation. Instead, we focus on verifying that the permutation does come after the original but we could not verify that there are no other permutations in between.

Lines 6-17: This corresponds to the functionality on lines 1013 of Figure 2. The addition of the loop invariants on lines 10 13 supports the verification of the post-conditions on lines $3-5$. In particular, the invariant on lines $11-12$ is necessary to prove the post-condition on line 4 corresponding to Task 1.4. The decreases clause on line 14 is provided to prove loop termination and contributes to the fulfilment of Task 1.2.

Lines 18-29: This corresponds to the functionality of lines 1418 of Figure 2. The loop invariants on lines 24-25 ensure the correct functioning of the loop with the invariant on line 24 concerned with memory safety (Task 1.1 ). The decreases clause on line 26 serves to prove termination (Task 1.2).

Lines 30-47: These lines correspond to lines 19-31 of Figure 2. The ghost variable on line 32 is used in the loop invariant on lines 37-38 which supports the verification of the postcondition on line 5 corresponding (partially) to Task 1.5 . Similarly, the invariant on line 39 supports the verification of the post-condition on line 3 (Task 1.3).
Lines 48-54: The permut method is specified by the pre-and post-conditions on lines 50-54. Memory safety (Task 1.6) is addressed by the modifies clause on line 50 . The postconditions on lines 51-53 ensure that all produced sequences are the same size and are in lexicographic order (Tasks 1.8 and 1.10), respectively. For Task 1.8, we get uniqueness from strict monotonicity, a strictly increasing sequence contains no duplicates.

Lines 55-83: These lines correspond to the functionality of lines 1-8 in Figure 2. We define a ghost variable (line 64) which is used by the invariant on lines $70-71$. This is used to prove that the pre-condition for 1 t seq is not violated when it is called in the invariant on lines 74-75. This invariant on lines $74-75$ is also needed to verify the invariant on line 76 which, in turn, supports the verification of the post-condition on line 53 (Tasks 1.8 and 1.10). The decreases clause on line 77 serves to prove termination (Task 1.7).

Overall, we were able to verify Tasks 1.1, 1.2, 1.3, 1.4, 1.6, 1.7, 1.8 and 1.10 . We partially verified Task 1.5 but we did not provide a particularly elegant (or complete) solution to Task 1.9 because we did not have a nice way of specifying that all permutations were reported. This could be a limitation of us as Dafny users or it could be due to the way that we constructed the algorithm.

For Task 1.9, our approach was to use the fact that, for a sequence of size $n$, there will be at most $n$ ! permutations. We thus included the loop variable fac on line 67 of Figure 4 and our loop condition enforces that next is not called more than fac times. Without this variable we could not prove that the loop would terminate or that next was only called the correct number of times.

With respect to Task 1.5, we were not able to completely verify that the proper next permutation is returned. Specifically, the post-condition that we were trying to verify was that there are no such sequences in between the one that has been returned and the previous one. This kind of property was difficult to verify in Dafny.

\subsection{Challenge 2: DLL to BST}

"This challenge is to verify an in-place algorithm to convert a sorted doubly-linked list (DLL) into a balanced binary search tree (BST)."

Figure 5 contains the corresponding pseudo code that was given to competitors. The verification tasks were as follows:

Task 2.1: Prove that this algorithm converts an input list into a tree.

Task 2.2: Prove that the algorithm is memory-safe.

Task 2.3: Prove that if the input list is sorted then the resulting tree is a BST.

Task 2.4: Prove that the resulting BST is balanced.

Task 2.5: Prove that the algorithm terminates.

Task 2.6: (Optional) Prove the above for an iterative version of size.

We were less successful with this challenge than we were with the previous one because we spent a lot of time encoding predicates to determine the validity of a binary search tree and doubly linked list. These predicates and a necessary constructor are contained in 


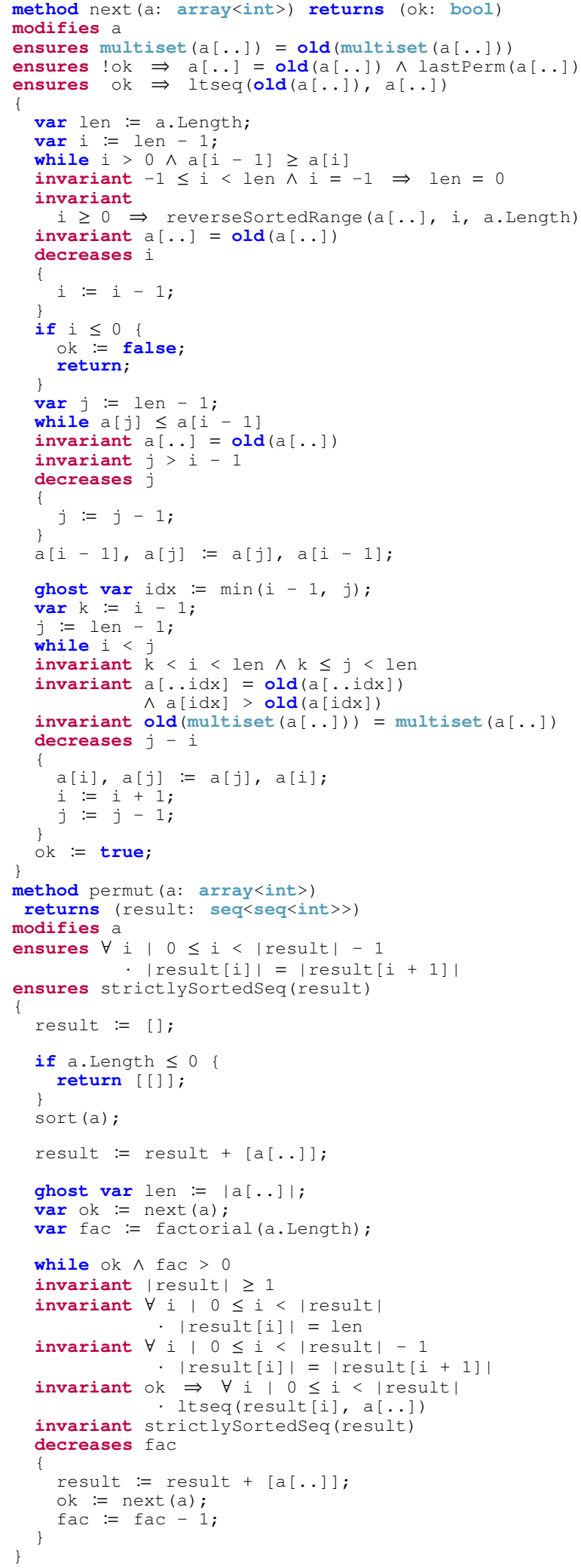

Figure 4: Dafny implementation of next (lines 1-47) and permut (lines 48-83).

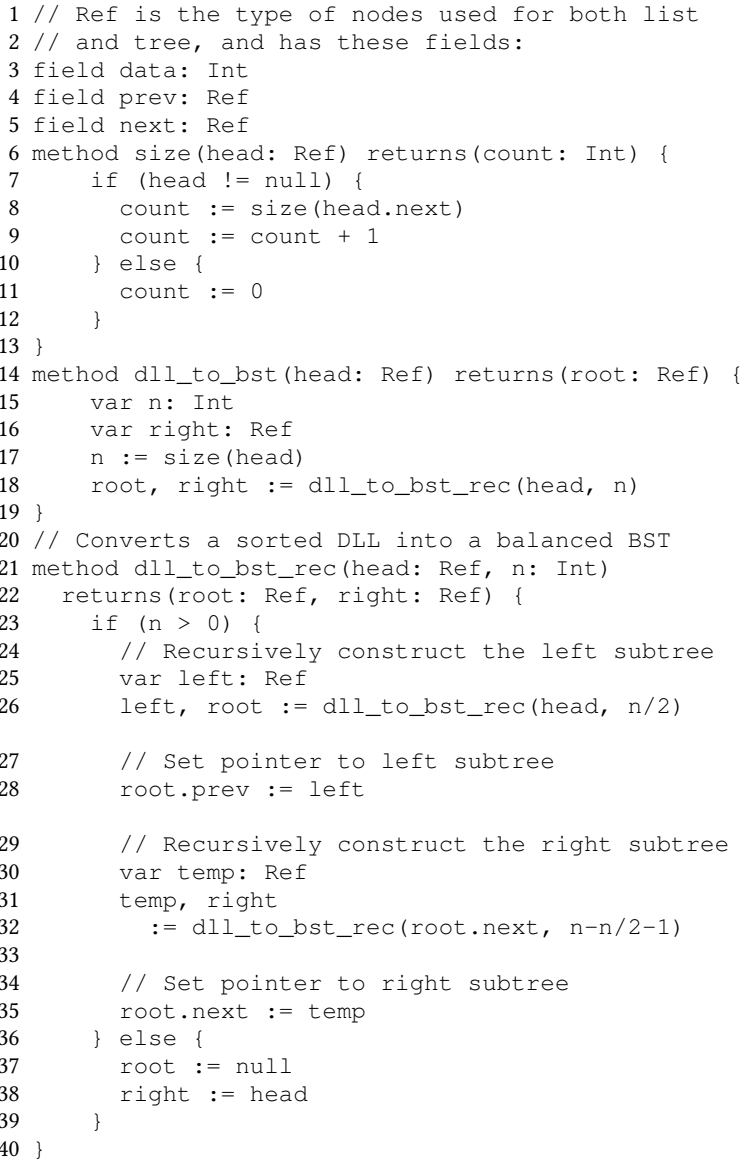

Figure 5: This pseudo code was given to the competitors as part of the Challenge 2 description.

Figure 6 which we describe below. We broadly followed a specification of binary trees that was available in the Dafny repository ${ }^{5}$.

Lines 1-5: This class describes the basic components of a variable of type Ref. This corresponds to the field definitions on lines $1-5$ of Figure 5 that was given to competitors.

Lines 6-8: We define two ghost variables to be used later. It is common when creating classes in Dafny to include both non-ghost and ghost variables. Here, the ghost variable Contents provides an abstract representation of the linked list for specification purposes. The set Repr contains all objects making up the representation set of a Ref, including itself and its subobjects. This is mainly used to describe frame conditions (the set of objects in reads and modifies clauses). For example, requiring that the sets prev. Repr and next. Repr are disjoint, using the '!!' operator, ensures that no object in the left subtree also appears in the right subtree, and vice versa. This also ensures that there are no cycles. We furthermore require that the left and right subtrees are 'subsets' of their parent, using the ' $<=$ ' operator. We also prove termination by showing that

$\overline{{ }^{5} \text { https://git.io/JG2i7 }}$ 


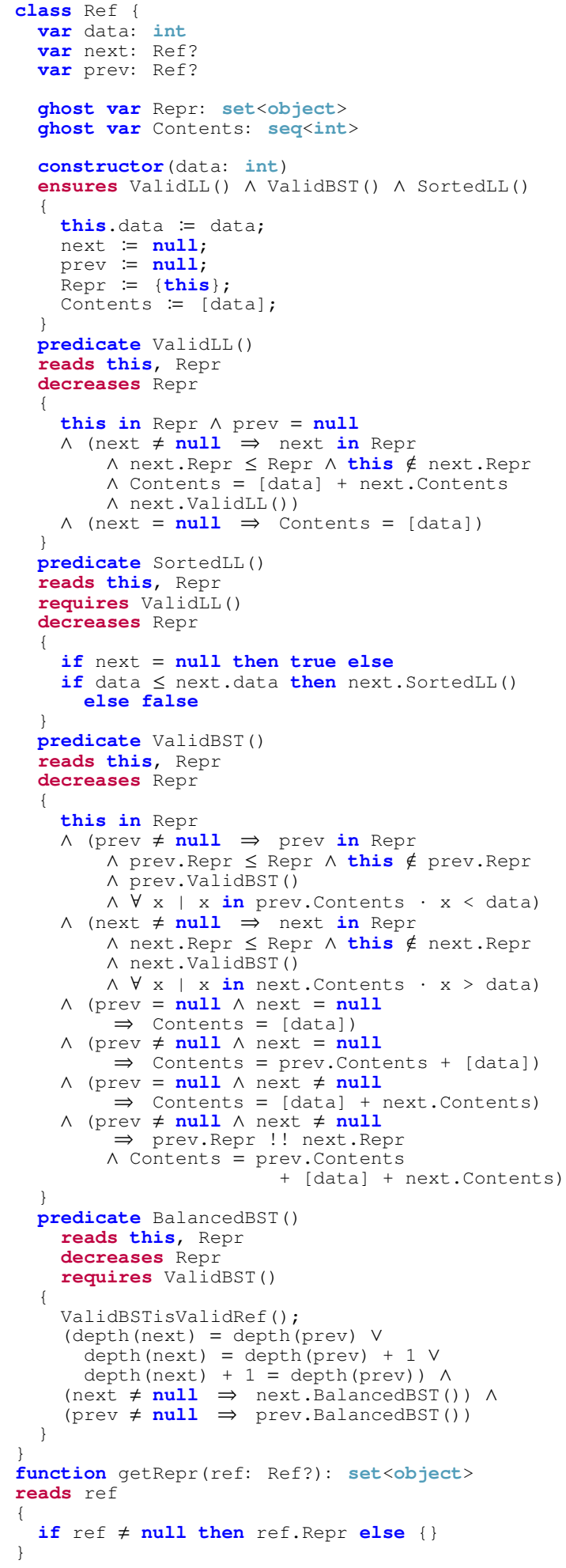

Figure 6: Predicates and Ref class in Dafny for Challenge 2.

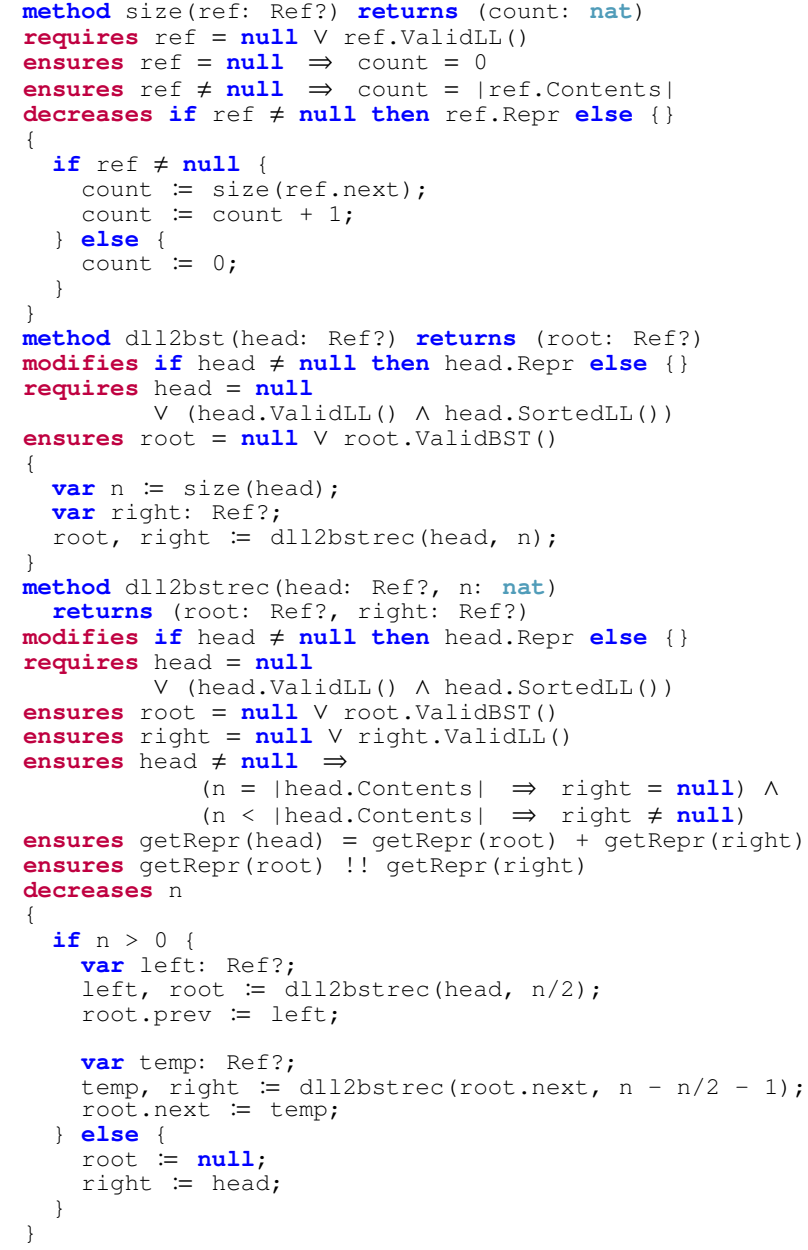

Figure 7: Dafny implementation for Challenge 2.

the size of Repr decreases with each recursive call (lines 20, 32, 40 and 64 of Figure 6).

Lines 9-17: Here we provide a constructor for this class.

Lines 18-28: This predicate specifies what it means to be a valid linked list. The reads clause on line 19 specifies the memory locations that the predicate is allowed to access. We use the decreases clause on line 20 to prove termination in the usual way. Note that we do not actually require that the linked list is a true doubly-linked list, merely that it is a valid singly-linked list, and that the reference prev exists.

Lines 29-37: This predicate specifies what it means to be a sorted linked list.

Lines 38-61: Here, we specify what it means for a binary search tree to be valid. In particular, the prev and next subtrees should also be valid binary search trees (lines 45 and 49 respectively). The prev and next subtrees should also be disjoint indicated by the '! !' operator on line 58 .

Lines 62-74: The ValidRef predicate is the same as the ValidBST predicate, but omits the requirement for left 
subtrees to contain smaller data, and right subtrees to contain larger data. It is our least specific requirement, but we still enforce that there are no cycles, since termination would otherwise be very difficult to determine in general.

Lines 75-79: This function is used to collect the set of representative objects for a given Ref.

The predicates defined in Figure 6 are necessary to verify Tasks 2.3 and 2.4. Our Dafny implementation of the functionality that was provided to competitors (shown in Figure 5) is contained in Figure 7 and we describe it as follows:

Lines 1-13: This is our Dafny implementation of the size function that was given on lines 6-13 of Figure 7. The precondition on line 2 requires that the input ref is either null or it is a valid linked list. We then ensure (line 3 ) that if ref is null then count will be 0 . Otherwise, it returns the size of ref (line 4). The decreases clause on line 5 is used to prove termination, which supports Task 2.5. In the case that ref is not null, the set of objects making up the representation of ref decreases in size, however if ref is null, then there is no such set, so we simply say that the empty set decreases.

Lines 14-23: This corresponds to lines 14-19 of the pseudo code that was given in Figure 5. We added the specifications on lines 15-18 for verification. The modifies clause is needed here and supports memory safety as prescribed by Task 2.2. We ensure (line 18) that the output is a valid binary search tree using the predicate that was previously defined. We note that these specifications were verified for this method, however, they depend on those in the subsequent method which we could not completely verify.

Lines 24-50: Here, we capture the main functionality of the algorithm, corresponding to lines $20-40$ of Figure 5. We were able to verify termination of this method (line 35 and Task $2.5)$. We verified memory safety via the modifies clause on line 26. We verified the post-conditions on lines 31-34 which offers some evidence towards Task 2.4. However, we encountered difficulties when trying to verify the specifications on lines 29-30. Much of the difficulty revolves around verifying that we have permission to stitch left and temp onto root, and deciding what exactly we can say about the resulting data structure.

We were able to completely verify Task 2.5 but not the other tasks. As a result, we did not attempt the optional Task 2.6.

A significant amount of time was spent trying to formalise a predicate describing a valid doubly-linked list. Termination of such a predicate follows from the fact that Repr, the set of objects making up the representation of a Ref is finite, and that it decreases in size with each call. This is not so simple with a doubly-linked list, since, as presented, there is no easy way to prove that such a predicate terminates. This stems from the difficulty in navigating a doubly linked list and knowing your exact location in the list at any given time. We might have had a better result if we had used another data structure to represent the list, for example a pair of stacks, and defined an invariant to describe the relationship between this and the doubly linked list, giving us more visibility of how we traverse the structure and providing more explicit termination measures. However, this may not have solved all of our problems.

Further, the use of potentially null variables and the recursive nature of the method on lines 24-50 of Figure 7 caused us problems with respect to proving the frame condition (modifies clause). We specify that head. Repr may be modified (line 15), which means that Dafny can modify any object in head. Repr. However, Dafny does not recognise that it is allowed to modify root (on line 41), despite the fact that Dafny knows that root is not null, that root is in head. Repr, and that it can modify any object in head.Repr, from which it should follow immediately that root is modifiable. In the discussion session at VerifyThis 2021 other teams presented similar issues and some managed to solve this with their verification tools. We also noted that at least one of the other teams participating in the competition using Dafny switched to using Isabelle for Challenge 2 .

\section{DISCUSSION}

The challenges in VerifyThis 2021 exposed a number of strengths and limitations of Dafny. Particular strengths included Dafny's ability to prove termination and memory safety with little input, especially in Challenge 1 (Tasks 1.1, 1.2. 1.6, 1.7 and 2.5). Dafny's built-in value types, i.e. sets, sequences, multisets, and maps, support ergonomic specification and verification. The multiset datatype was particularly useful for verification of Challenge 1, allowing us to state and easily prove that two arrays contained the same elements without needing to write additional functions or predicates to verify Task 1.3. Likewise, we used Dafny's immutable sequence types seq when specifying both challenges, and the set type in Challenge 2 for tracking the representation of an object.

We used two main constructs to support the specification of aggregate objects in Challenge 2. These are object invariants, which we express as a validity predicate e.g. ValidLL (lines 18-28 of Figure 6), and a representation set, expressed as a ghost variable Repr (line 15 of Figure 6) representing the object's dynamic frame ${ }^{6}$. This representation of an object's dynamic frame is provided as a set of objects, so Repr can contain references to objects of any type and that set of objects can dynamically change over time. We can specify permissions on this set, using reads and modifies clauses, specified for each predicate, function or method provided.

Dafny's predicates and lemmas helped us with verification tasks throughout the challenges, allowing us to make our specifications more concise. Dafny provides automatic induction proofs for lemmas which we found useful in our proofs. We also took advantage of Dafny's specification-only ghost constructs in both Challenge 1 , for verification of loop invariants, and Challenge 2, for the abstract representation of the linked list and set of representation objects as mentioned above.

It was difficult to verify properties about possibly null objects in Challenge 2 e.g. lines 24-50 of Figure 7. A larger variety of built-in datatypes would potentially have been useful here. The decreases annotation provides an expression that decreases with every recursive call and is bounded below. If a recursive function or method is not given an explicit decreases clause, then

\footnotetext{
${ }^{6}$ The name of the programming language Dafny comes from a permutation of the letters in "dynamic frames".
} 
the Dafny verifier guesses one based on the parameter provided by the function or method. This was useful apart from when more sophisticated measures were required, e.g. line 5 of Figure 5. Further to this, the error messages provided by Dafny were often not informative, but this is a limitation of many verification tools.

A nice aspect of Dafny is that memory safety is verified without a large specification overhead. For Challenge 1, array bounds are verified via the associated variants and invariants. The use of modifies clauses allow us to specify which memory locations are allowed to be modified by the relevant methods. If no modifies clause is given then the default is that no memory locations can be modified. These modifies clauses are accompanied by reads clauses in Challenge 2 to control which memory locations are accessible (e.g. the current object and its representation on lines 19 , 30, 39 and 63 of Figure 6).

The competition provided both a challenge to the tool and to the users of the tool. It was beneficial for us, the users, because it allowed us to learn more about Dafny as well as examine how Dafny performs when verifying relatively complex algorithmic problems. To date we have not seen details of the other Dafny solutions submitted to VerifyThis 2021 but we look forward to comparing our efforts when they are made available. Likewise, a deeper discussion with other teams, regarding how they used their tool to solve the challenges would be interesting. We hope to resolve the problems that we encountered and we thank K. Rustan. M. Leino for preliminary discussions regarding potential solutions.

\section{CONCLUSIONS}

Participation in the VerifyThis 2021 competition was educational because it allowed us to examine the usability and usefulness of Dafny for algorithmic verification tasks. It also gave us an opportunity to learn more about the tool and gave us a better understanding of its strengths and weaknesses. We would like to offer congratulations to the prize winners of VerifyThis 2021. VerifyThis 2021 was sponsored by Amazon (AWS) and the winners are listed at: https://www.pm.inf.ethz.ch/research/verifythis/Prizes.html.

\section{REFERENCES}

[1] Mike Barnett, Bor-Yuh Evan Chang, Robert DeLine, Bart Jacobs, and K Rustan M Leino. 2005. Boogie: A modular reusable verifier for object-oriented programs. In
Formal Methods for Components and Objects (LNCS, Vol. 4111). Springer, 364-387. https://doi.org/10.1007/11804192_17

[2] Thorsten Bormer, Marc Brockschmidt, Dino Distefano, Gidon Ernst, JeanChristophe Filliâtre, Radu Grigore, Marieke Huisman, Vladimir Klebanov, Claude Marché, Rosemary Monahan, Wojciech Mostowski, Nadia Polikarpova, Christoph Scheben, Gerhard Schellhorn, Bogdan Tofan, Julian Tschannen, and Mattias Ulbrich. 2012. The COST IC0701 Verification Competition 2011. In Formal Verification of Object-Oriented Software (LNCS, Vol. 7421). Springer, 3-21. https: //doi.org/10.1007/978-3-642-31762-0_2

[3] Leonardo De Moura and Nikolaj Bjørner. 2008. Z3: An efficient SMT solver. In Tools and Algorithms for the Construction and Analysis of Systems (LNCS, Vol. 4963). Springer, 337-340. https://doi.org/10.1007/978-3-540-78800-3_24

[4] Claire Dross, Carlo A Furia, Marieke Huisman, Rosemary Monahan, and Peter Müller. 2021. VerifyThis 2019: a program verification competition. International Journal on Software Tools for Technology Transfer (2021), 1-11. https://doi.org/10. 1007/s10009-021-00619-x

[5] Marie Farrell, Matthew Bradbury, Michael Fisher, Louise A Dennis, Clare Dixon, Hu Yuan, and Carsten Maple. 2019. Using Threat Tnalysis Techniques to Guide Formal Verification: A Case Study of Cooperative Awareness Messages. In International Conference on Software Engineering and Formal Methods (LNCS, Vol. 11724). Springer, 471-490. https://doi.org/10.1007/978-3-030-30446-1_25

[6] Marie Farrell, Nikos Mavrakis, Clare Dixon, and Yang Gao. 2020. Formal Verification of an Autonomous Grasping Algorithm. In International Symposium on Artificial Intelligence, Robotics and Automation in Space. ESA.

[7] Marieke Huisman, Vladimir Klebanov, and Rosemary Monahan. 2015. VerifyThis 2012: A Program Verification Competition. International fournal on Software Tools for Technology Transfer 17 (2015), 647-657. https://doi.org/10.1007/s10009015-0396-8

[8] Marieke Huisman, Vladimir Klebanov, Rosemary Monahan, and Michael Tautschnig. 2017. VerifyThis 2015. International fournal on Software Tools for Technology Transfer 19, 6 (2017), 763-771. https://doi.org/10.1007/s10009-016-0438-x

[9] Marieke Huisman, Rosemary Monahan, Peter Müller, Wojciech Mostowski, and Mattias Ulbrich. 2017. VerifyThis 2017: A Program Verification Competition. Number 2017-10 in Karlsruhe Reports in Informatics. Karlsruhe Institute of Technology.

[10] Marieke Huisman, Rosemary Monahan, Peter Müller, Andrei Paskevich, and Gidon Ernst. 2019. VerifyThis 2018: A Program Verification Competition. Research Report. Université Paris-Saclay.

[11] Marieke Huisman, Rosemary Monahan, Peter Muller, and Erik Poll. 2016. VerifyThis 2016: A program verification competition. (2016).

[12] Vladimir Klebanov, Peter Müller, Natarajan Shankar, Gary T. Leavens, Valentin Wüstholz, Eyad Alkassar, Rob Arthan, Derek Bronish, Rod Chapman, Ernie Cohen, Mark Hillebrand, Bart Jacobs, K. Rustan M. Leino, Rosemary Monahan, Frank Piessens, Nadia Polikarpova, Tom Ridge, Jan Smans, Stephan Tobies, Thomas Tuerk, Mattias Ulbrich, and Benjamin Weiß. 2011. The 1st Verified Software Competition: Experience Report. In International Symposium on Formal Methods (LNCS, Vol. 6664). Springer, 154-168. https://doi.org/10.1007/978-3-642-21437$0 \_14$

[13] Rafael Krucker and Markus Schaden. 2017. Visual Studio Code Integration for the Dafny Language and Program Verifier. Ph.D. Dissertation. HSR Hochschule für Technik Rapperswil.

[14] K Rustan M Leino. 2010. Dafny: An automatic program verifier for functional correctness. In International Conference on Logic for Programming Artificial Intelligence and Reasoning (LNCS, Vol. 6355). Springer, 348-370. https: //doi.org/10.1007/978-3-642-17511-4_20 